\title{
Recovery of Missing Samples from Multi-Channel Oversampling in Shift-Invariant Spaces
}

\author{
Sinuk Kang \\ Laboratory of Mathematics Applied to Systems, Ecole Centrale Paris \\ Grande voie de vignes, 92290 Châtenay Malabry, France \\ sinuk.kang@ecp.fr \\ Kil Hyun Kwon \\ Department of Mathematical Sciences, KAIST \\ Daejeon, 305-701, Republic of Korea \\ khkwon@kaist.edu \\ Dae Gwan Lee \\ Department of Mathematical Sciences, KAIST \\ Daejeon, 305-701, Republic of Korea \\ daegwan@kaist.ac.kr
}

\begin{abstract}
It is well known that in the classical Shannon sampling theory on bandlimited signals, any finitely many missing samples can be recovered when the signal is oversampled at a rate higher than the minimum Nyquist rate. In this work, we consider the problem of recovering missing samples from multi-channel oversampling in a general shift-invariant space. We find conditions under which any finite or infinite number of missing samples can be recovered when they are from a single or two channels.
\end{abstract}

Key words and phrases : recovery of missing samples, shift-invariant space, oversampling, multi-channel sampling, frame

2000 AMS Mathematics Subject Classification — 42C15, 94A20

\section{Introduction}

Any band-limited signal can be reconstructed using its equidistant sample values due to the classical Shannon sampling theorem [7]. When a band-limited signal is sampled at the minimum Nyquist rate, in general, perfect reconstruction fails even if a single sample value is missing. However, if it is sampled at a rate 
higher than the minimum Nyquist rate (oversampling), then the sample values thus obtained have some redundancy which enables perfect recovery even when finitely many samples are missing $[3,4,11]$. Adapting these ideas to two-channel oversampling involving the signal and its derivative, Santos and Ferreira [13] showed that any finite missing samples can be recovered if they belong to a single channel (see also [1, 2]). Most works on this problem are for the bandlimited signals or for Kramer's sampling expansion [3]. Only recently, has it been considered in [8] for multi-channel oversampling expansion of signals in a principal shift-invariant space with a Riesz generator with compact support when all the reconstruction functions also have compact support.

In this paper, as an extension of the results in [8], we consider the recovery of missing samples for the multi-channel oversampling expansion on a principal shift-invariant space without any compact supportedness of its generator or reconstruction functions. We find sufficient conditions for the recovery of a finite or infinite number of samples missing from a single channel or from two channels.

\section{Preliminaries}

On $L^{2}(\mathbb{R}) \cap L^{1}(\mathbb{R})$, we take the Fourier transform to be normalized as

$$
\mathcal{F}[f](\xi)=\hat{f}(\xi):=\int_{-\infty}^{\infty} f(t) e^{-i t \xi} d t, \quad f(t) \in L^{2}(\mathbb{R}) \cap L^{1}(\mathbb{R})
$$

so that $\frac{1}{\sqrt{2 \pi}} \mathcal{F}[\cdot]$ becomes a unitary operator from $L^{2}(\mathbb{R})$ onto $L^{2}(\mathbb{R})$. For any $\phi(t) \in L^{2}(\mathbb{R})$, let

$$
C_{\phi}(t):=\sum_{n \in \mathbb{Z}}|\phi(t+n)|^{2} \quad \text { and } \quad G_{\phi}(\xi):=\sum_{n \in \mathbb{Z}}|\hat{\phi}(\xi+2 n \pi)|^{2} .
$$

Then $C_{\phi}(t)=C_{\phi}(t+1) \in L^{1}[0,1], G_{\phi}(\xi)=G_{\phi}(\xi+2 \pi) \in L^{1}[0,2 \pi]$ and

$$
\|\phi(t)\|_{L^{2}(\mathbb{R})}^{2}=\left\|C_{\phi}(t)\right\|_{L^{1}[0,1]}=\frac{1}{2 \pi}\left\|G_{\phi}(\xi)\right\|_{L^{1}[0,2 \pi]} .
$$

We also let

$$
Z_{\phi}(t, \xi):=\sum_{n \in \mathbb{Z}} \phi(t+n) e^{-i n \xi}
$$

be the Zak transform of $\phi(t) \in L^{2}(\mathbb{R})$. Then $Z_{\phi}(t, \xi) \in L^{2}([0,1] \times[0,2 \pi])$. For any $2 \pi$-periodic function $\tau(\xi)$, let $\|\tau(\xi)\|_{0}$ and $\|\tau(\xi)\|_{\infty}$ be the essential infimum and the essential supremum of $|\tau(\xi)|$ on $[0,2 \pi]$ respectively.

For any $\mathbf{c}=\{c(n)\}_{n \in \mathbb{Z}}$ in $l^{2}(\mathbb{Z})$, let

$$
\mathcal{F}[\mathbf{c}](\xi)=\hat{\mathbf{c}}(\xi):=\sum_{n \in \mathbb{Z}} c(n) e^{-i n \xi}
$$


be the discrete Fourier transform of $\mathbf{c}$. Then $\hat{\mathbf{c}}(\xi)=\hat{\mathbf{c}}(\xi+2 \pi) \in L^{2}[0,2 \pi]$ and

$$
\|\mathbf{c}\|_{l^{2}(\mathbb{Z})}^{2}:=\sum_{n \in \mathbb{Z}}|c(n)|^{2}=\frac{1}{2 \pi}\|\hat{\mathbf{c}}(\xi)\|_{L^{2}[0,2 \pi]}^{2}
$$

For any $\mathbf{c}=\{c(n)\}_{n \in \mathbb{Z}}$ and $\mathbf{d}=\{d(n)\}_{n \in \mathbb{Z}}$ in $l^{2}(\mathbb{Z})$, let

$$
(\mathbf{c} * \mathbf{d})(n):=\sum_{k \in \mathbb{Z}} c(k) d(n-k)
$$

be the discrete convolution product of $\mathbf{c}$ and $\mathbf{d}$. Then $\mathbf{c} * \mathbf{d} \in l^{\infty}(\mathbb{Z})$ and $\widehat{\mathbf{c} * \mathbf{d}}(\xi)=\hat{\mathbf{c}}(\xi) \hat{\mathbf{d}}(\xi)$ whenever $\hat{\mathbf{c}}(\xi) \hat{\mathbf{d}}(\xi) \in L^{2}[0,2 \pi]$ or $\mathbf{c} * \mathbf{d} \in l^{2}(\mathbb{Z})$.

A Hilbert space $H$ consisting of complex valued functions on a set $E$ is called a reproducing kernel Hilbert space (RKHS) if there is a function $q(s, t)$ on $E \times E$, called the reproducing kernel of $H$, satisfying

- $q(\cdot, t) \in H$ for each $t$ in $E$;

- $\langle f(s), q(s, t)\rangle=f(t), f \in H$.

In an RKHS $H$, any norm converging sequence also converges uniformly on any subset of $E$, on which $q(t, t)$ is bounded (Section 3.4 in [7]).

For any $\phi \in L^{2}(\mathbb{R})$, let $V(\phi):=\overline{\operatorname{span}}\{\phi(t-n): n \in \mathbb{Z}\}$ be the shift-invariant space generated by $\phi$. We call $\phi$ a Riesz generator (resp. frame generator) when $\{\phi(t-n): n \in \mathbb{Z}\}$ is a Riesz basis (resp. frame) of $V(\phi)$.

\section{Main Results}

In this work, we always assume that $\phi(t) \in L^{2}(\mathbb{R}) \cap C(\mathbb{R})$ is a continuous Riesz generator satisfying $\sup _{\mathbb{R}} C_{\phi}(t)<\infty$. Then

$$
V(\phi)=\left\{(\mathbf{c} * \phi)(t)=\sum_{n \in \mathbb{Z}} c(n) \phi(t-n): \mathbf{c} \in l^{2}(\mathbb{Z})\right\} \subset L^{2}(\mathbb{R}) \cap C(\mathbb{R})
$$

is a reproducing kernel Hilbert space and for any $\mathbf{c} \in l^{2}(\mathbb{Z}),(\mathbf{c} * \phi)(t)=$ $\sum_{n \in \mathbb{Z}} c_{n} \phi(t-n)$ converges both in $L^{2}(\mathbb{R})$ and absolutely and uniformly on $\mathbb{R}$ to a bounded continuous function. Moreover, the reproducing kernel of $V(\phi)$ is

$$
q(s, t):=\sum_{n \in \mathbb{Z}} \overline{\phi(t-n)} \tilde{\phi}(s-n)
$$

where $\{\tilde{\phi}(t-n)\}_{n \in \mathbb{Z}}$ is the dual Riesz basis of $\{\phi(t-n)\}_{n \in \mathbb{Z}}$. Since $\sup _{\mathbb{R}} C_{\phi}(t)<$ $\infty$, the function $q(t, t)$ is bounded on $\mathbb{R}$. 
Let $\mathcal{L}[\cdot]$ be an LTI (linear time-invariant) system with impulse response $l(t)$, that is,

$$
\mathcal{L}[f](t):=(l * f)(t)=\int_{\mathbb{R}} l(t-s) f(s) d s .
$$

Here we assume that $l(t)$ is one of the following three types:

(i) $l(t)=\delta(t+a), a \in \mathbb{R}$;

(ii) $l(t) \in L^{2}(\mathbb{R})$;

(iii) $l(t) \in L^{1}(\mathbb{R})$.

Lemma 3.1. Let $\mathcal{L}[\cdot]$ be an LTI system with impulse response $l(t)$ as above and $\psi(t):=\mathcal{L}[\phi](t)=(l * \phi)(t)$. Then

(a) $\psi(t) \in C(\mathbb{R})$ and $\sup _{\mathbb{R}} C_{\psi}(t)<\infty$.

(b) For any $f(t)=(\mathbf{c} * \phi)(t) \in V(\phi)$ with $\mathbf{c} \in l^{2}(\mathbb{Z}), \mathcal{L}[f](t)=(\mathbf{c} * \psi)(t)$ converges absolutely and uniformly on $\mathbb{R}$. Hence $\mathcal{L}[f](t) \in C(\mathbb{R})$.

Proof. For types (i) and (ii), see the proof of Lemma 3.1 in [5] or Lemma 3.1 in [9].

Assume that $l(t) \in L^{1}(\mathbb{R})$. Then for any $t$ and $t_{0}$ in $\mathbb{R},\left|\psi(t)-\psi\left(t_{0}\right)\right| \leq$ $\|\phi\|_{L^{\infty}(\mathbb{R})} \int_{\mathbb{R}}\left|l(t-s)-l\left(t_{0}-s\right)\right| d s \rightarrow 0$ as $t \rightarrow t_{0}$. Hence $\psi(t) \in C(\mathbb{R})$. Since $|\psi(t)| \leq \int_{\mathbb{R}}|l(s)|^{\frac{1}{2}}|l(s)|^{\frac{1}{2}}|\phi(t-s)| d s \leq\left(\int_{\mathbb{R}}|l(s)| d s\right)^{\frac{1}{2}}\left(\int_{\mathbb{R}}|l(s)||\phi(t-s)|^{2} d s\right)^{\frac{1}{2}}$, we have

$$
\sum_{n \in \mathbb{Z}}|\psi(t+n)|^{2} \leq\|l\|_{L^{1}(\mathbb{R})} \int_{\mathbb{R}}|l(s)| \sum_{n \in \mathbb{Z}}|\phi(t+n-s)|^{2} d s \leq\|l\|_{L^{1}(\mathbb{R})}^{2} \sup _{\mathbb{R}} C_{\phi}(t)
$$

so that $\sup _{\mathbb{R}} C_{\psi}(t)<\infty$. By Young's inequality, $\mathcal{L}[\cdot]: L^{2}(\mathbb{R}) \rightarrow L^{2}(\mathbb{R})$ is a bounded linear operator so that, for any $f(t)=(\mathbf{c} * \phi)(t) \in V(\phi), \mathcal{L}[f](t)=$ $\sum_{n \in \mathbb{Z}} c(n) \mathcal{L}[\phi(t-n)]=\sum_{n \in \mathbb{Z}} c(n) \psi(t-n)=(\mathbf{c} * \psi)(t)$ converges in $L^{2}(\mathbb{R})$. Then (a) implies that $(\mathbf{c} * \psi)(t)$ also converges absolutely and uniformly on $\mathbb{R}$ to $\mathcal{L}[f](t) \in C(\mathbb{R})$.

For later use, we note that $\mathcal{L}[\cdot]$ is a bounded linear operator from $L^{2}(\mathbb{R})$ into $L^{2}(\mathbb{R})$ for types (i) and (iii) and a bounded linear operator from $L^{2}(\mathbb{R})$ into $L^{\infty}(\mathbb{R})$ for type (ii).

Let $\left\{\mathcal{L}_{j}[\cdot]: 1 \leq j \leq N\right\}$ be $N$ LTI systems with impulse responses $\left\{l_{j}(t)\right.$ : $1 \leq j \leq N\}$ where each $l_{j}(t)$ is one of the above three types. We are interested in the recovery of missing samples for the multi-channel oversampling expansion:

$$
f(t)=\sum_{j=1}^{N} \sum_{n \in \mathbb{Z}} \mathcal{L}_{j}[f]\left(\sigma_{j}+n r\right) S_{j}(t-n r), \quad f \in V(\phi),
$$


where $\sigma_{j} \in \mathbb{R}$ for $1 \leq j \leq N, r$ is a positive integer, and $\left\{S_{j}(t-n r): 1 \leq j \leq\right.$ $N, n \in \mathbb{Z}\}$ is a frame of $V(\phi)$. Before we discuss our main problem, we first need to recall conditions under which such a multi-channel oversampling expansion is possible on $V(\phi)$.

Let $\mathcal{D}$ be the unitary operator from $L^{2}[0,2 \pi]$ onto $L^{2}\left[0, \frac{2 \pi}{r}\right]^{r}$ defined as

$$
\mathcal{D} F:=\left[F\left(\xi+(k-1) \frac{2 \pi}{r}\right)\right]_{k=1}^{r}, \quad F \in L^{2}[0,2 \pi]
$$

and

$$
G(\xi):=\left[\mathcal{D} g_{1}, \ldots, \mathcal{D} g_{N}\right]^{T}=\left[g_{j}\left(\xi+(k-1) \frac{2 \pi}{r}\right)\right]_{1 \leq j \leq N, 1 \leq k \leq r}
$$

be an $N \times r$ matrix where $g_{j}(\xi):=\frac{1}{2 \pi} Z_{\psi_{j}}\left(\sigma_{j}, \xi\right)$ and $\psi_{j}(t):=\mathcal{L}_{j}[\phi](t)$. Let $\alpha_{G}:=$ $\left\|\lambda_{\min }\left(G(\xi)^{*} G(\xi)\right)\right\|_{0}$ and $\beta_{G}:=\left\|\lambda_{\max }\left(G(\xi)^{*} G(\xi)\right)\right\|_{\infty}$ where $\lambda_{\min }\left(G(\xi)^{*} G(\xi)\right)$ and $\lambda_{\max }\left(G(\xi)^{*} G(\xi)\right)$ are the smallest and the largest eigenvalues of the positive semi-definite $r \times r$ matrix $G(\xi)^{*} G(\xi)$, respectively.

Proposition 3.2 (see Theorem 2 and Corollary 1 in [6] or Theorem 3.3 and Theorem 3.4 in [9]). Assume $\beta_{G}<\infty$, i.e., $Z_{\psi_{j}}\left(\sigma_{j}, \xi\right) \in L^{\infty}[0,2 \pi], 1 \leq j \leq N$. Then the following are equivalent.

(a) There is a frame $\left\{S_{j}(t-n r): 1 \leq j \leq N, n \in \mathbb{Z}\right\}$ of $V(\phi)$ such that

$$
f(t)=\sum_{j=1}^{N} \sum_{n \in \mathbb{Z}} \mathcal{L}_{j}[f]\left(\sigma_{j}+n r\right) S_{j}(t-n r), \quad f \in V(\phi) .
$$

(b) $\alpha_{G}>0$.

Moreover we have in this case:

- series (1) converges both in $L^{2}(\mathbb{R})$ and absolutely and uniformly on $\mathbb{R}$;

- $r \leq N$;

- $r=N$ if and only if $\left\{S_{j}(t-n r): 1 \leq j \leq N, n \in \mathbb{Z}\right\}$ is a Riesz basis of $V(\phi)$;

- $\left\{\mathcal{L}_{j}[f]\left(\sigma_{j}+n r\right)\right\}_{n \in \mathbb{Z}} \in l^{2}(\mathbb{Z})$ for $1 \leq j \leq N$.

Note that if $r=N$ and $0<\alpha_{G} \leq \beta_{G}<\infty$ so that $\left\{S_{j}(t-n r): 1 \leq j \leq\right.$ $N, n \in \mathbb{Z}\}$ is a Riesz basis of $V(\phi)$ for which (1) holds, then it is impossible to recover any set of missing samples from (1) due to the uniqueness of basis expansion.

Hence in the following, we assume $r<N$ and $0<\alpha_{G} \leq \beta_{G}<\infty$ so that the sampling expansion (1) holds for an overcomplete frame $\left\{S_{j}(t-n r): 1 \leq j \leq\right.$ $N, n \in \mathbb{Z}\}$ of $V(\phi)$. Notice that $\left\{S_{j}(t-n): 1 \leq j \leq N, n \in \mathbb{Z}\right\}$ is also a frame of $V(\phi)$ so that $0<\left\|\sum_{j=1}^{N} G_{S_{j}}(\xi)\right\|_{0} \leq\left\|\sum_{j=1}^{N} G_{S_{j}}(\xi)\right\|_{\infty}<\infty$ (see Theorem 
2.2.14 in [12]). Also $\sup _{\mathbb{R}} \sum_{j=1}^{N} C_{S_{j}}(t)<\infty$ since $\sum_{j=1}^{N} \sum_{n \in \mathbb{Z}}\left|S_{j}(t-n)\right|^{2}=$ $\sum_{j=1}^{N} \sum_{n \in \mathbb{Z}}\left|\left\langle S_{j}(\cdot-n), q(\cdot, t)\right\rangle_{L^{2}(\mathbb{R})}\right|^{2} \leq B\|q(\cdot, t)\|_{L^{2}(\mathbb{R})}=B q(t, t)$, where $B$ is an upper frame bound of $\left\{S_{j}(t-n): 1 \leq j \leq N, n \in \mathbb{Z}\right\}$.

Let $\mathcal{L}[\cdot]$ be any one of the prescribed LTI systems $\mathcal{L}_{j}[\cdot], 1 \leq j \leq N$. Then applying $\mathcal{L}[\cdot]$ to $(1)$ gives

$$
\mathcal{L}[f](t)=\sum_{j=1}^{N} \sum_{n \in \mathbb{Z}} \mathcal{L}_{j}[f]\left(\sigma_{j}+n r\right) \mathcal{L}\left[S_{j}\right](t-n r), \quad f \in V(\phi),
$$

which converges either in $L^{2}(\mathbb{R})$ or $L^{\infty}(\mathbb{R})$ by the continuity of $\mathcal{L}[\cdot]$. We claim that (2) also converges absolutely and uniformly on $\mathbb{R}$ to $\mathcal{L}[f](t) \in C(\mathbb{R})$. If $\mathcal{L}[\cdot]$ is of type (i), then the claim is trivial. For type (ii), using the Poisson summation formula (cf. Lemma 5.1 in [10]) we have

$$
\begin{aligned}
\sum_{j=1}^{N} \sum_{n \in \mathbb{Z}}\left|\mathcal{L}\left[S_{j}\right](t+n)\right|^{2} & =\frac{1}{2 \pi} \sum_{j=1}^{N}\left\|\sum_{n \in \mathbb{Z}} \mathcal{L}\left[S_{j}\right](t+n) e^{-i n \xi}\right\|_{L^{2}[0,2 \pi]}^{2} \\
& =\frac{1}{2 \pi} \sum_{j=1}^{N}\left\|\sum_{n \in \mathbb{Z}} \hat{l}(\xi+2 n \pi) \hat{S}_{j}(\xi+2 n \pi) e^{i t(\xi+2 n \pi)}\right\|_{L^{2}[0,2 \pi]}^{2} \\
& \leq \frac{1}{2 \pi} \sum_{j=1}^{N}\left\|G_{l}(\xi)^{\frac{1}{2}} G_{S_{j}}(\xi)^{\frac{1}{2}}\right\|_{L^{2}[0,2 \pi]}^{2} \\
& =\frac{1}{2 \pi} \int_{0}^{2 \pi} G_{l}(\xi) \sum_{j=1}^{N} G_{S_{j}}(\xi) d \xi \\
& \leq\left\|\sum_{j=1}^{N} G_{S_{j}}(\xi)\right\|_{\infty}\|l\|_{L^{2}(\mathbb{R})}<\infty
\end{aligned}
$$

For type (iii), observe that

$$
\begin{aligned}
\sum_{j=1}^{N} \sum_{n \in \mathbb{Z}}\left|\mathcal{L}\left[S_{j}\right](t+n)\right|^{2} & \leq \sum_{j=1}^{N} \sum_{n \in \mathbb{Z}}\left(\int_{\mathbb{R}}|l(s)|^{\frac{1}{2}}|l(s)|^{\frac{1}{2}}\left|S_{j}(t+n-s)\right| d s\right)^{2} \\
& \leq\|l\|_{L^{1}(\mathbb{R})} \sum_{j=1}^{N} \sum_{n \in \mathbb{Z}} \int_{\mathbb{R}}|l(s)|\left|S_{j}(t+n-s)\right|^{2} d s \\
& =\|l\|_{L^{1}(\mathbb{R})} \sum_{j=1}^{N} \int_{\mathbb{R}}|l(s)| C_{S_{j}}(t-s) d s \\
& \leq\|l\|_{L^{1}(\mathbb{R})}^{2} \sum_{j=1}^{N} \sup _{\mathbb{R}} C_{S_{j}}(t)<\infty .
\end{aligned}
$$


Hence $\sup _{\mathbb{R}} \sum_{j=1}^{N} \sum_{n \in \mathbb{Z}}\left|\mathcal{L}\left[S_{j}\right](t+n)\right|^{2}<\infty$ for both types (ii) and (iii). Since $\mathcal{L}\left[S_{j}\right](t) \in C(\mathbb{R}), 1 \leq j \leq N$ and $\left\{\mathcal{L}_{j}[f]\left(\sigma_{j}+n r\right): 1 \leq j \leq N, n \in \mathbb{Z}\right\} \in$ $l^{2}(\mathbb{Z})^{N}$, the right hand side of $(2)$ then converges absolutely and uniformly to a continuous function $\mathcal{L}[f](t)$ on $\mathbb{R}$.

We are now ready to consider the problem of recovering (finite or infinite) missing sample values among $\left\{\mathcal{L}_{j}[f]\left(\sigma_{j}+n r\right): 1 \leq j \leq N, n \in \mathbb{Z}\right\}$. In the following, let $\mathbf{B}_{j}$ and $\mathbf{B}^{k}$ be the $j$-th row and the $k$-th column of a matrix $\mathbf{B}=\left[b_{j, k}\right]_{j \in \mathbb{Z}, 1 \leq k \leq m}$ with infinite rows and finite columns. We view $\mathbf{B}^{k}$ both as a column vector and as a sequence $\left\{b_{j, k}\right\}_{j \in \mathbb{Z}}$. For any sequence $\mathbf{c}=\{c(n)\}_{n \in \mathbb{Z}}$ and $k \in \mathbb{Z}$, let $\tau_{k} \mathbf{c}=\{c(n-k)\}_{n \in \mathbb{Z}}$ be the shifting of $\mathbf{c}$ by $k$,

$$
\alpha(\mathbf{c}):=\inf \{n \in \mathbb{Z}: c(n) \neq 0\} \quad \text { and } \quad \beta(\mathbf{c}):=\sup \{n \in \mathbb{Z}: c(n) \neq 0\} .
$$

Then $\alpha\left(\tau_{k} \mathbf{c}\right)=\alpha(\mathbf{c})+k$ and $\beta\left(\tau_{k} \mathbf{c}\right)=\beta(\mathbf{c})+k$ for any $k \in \mathbb{Z}$. If $\mathbf{c} \neq 0$, then $-\infty \leq \alpha(\mathbf{c}) \leq \beta(\mathbf{c}) \leq \infty$, whereas $\alpha(\mathbf{0})=\infty$ and $\beta(\mathbf{0})=-\infty$. We say that $\mathbf{c}$ is upper (resp. lower) supported if $\alpha(\mathbf{c})$ (resp. $\beta(\mathbf{c})$ ) is finite. If $\mathbf{c}$ is either upper or lower supported, then we say that $\mathbf{c}$ is one-side supported. Let $\boldsymbol{\delta}_{0}:=\left\{\delta_{0, n}\right\}_{n \in \mathbb{Z}}$.

Case 1: finitely many missing samples from a single channel Assume that finitely many samples are missing from a single channel, say, from the first channel. That is, we assume that $M=\left\{\mathcal{L}_{1}[f]\left(\sigma_{1}+n r\right): n \in I\right\}$ is missing where $I=\left\{n_{1}<\cdots<n_{m}\right\}, m \geq 1$. Then we have from (2) with $\mathcal{L}=\mathcal{L}_{1}$ and $t=\sigma_{1}+\ell r$,

$$
\left.\mathcal{L}_{1}[f]\left(\sigma_{1}+\ell r\right)=\sum_{n \in \mathbb{Z}} \mathcal{L}_{1}[f]\left(\sigma_{1}+n r\right) \mathcal{L}_{1}\left[S_{1}\right]\left(\sigma_{1}+(\ell-n) r\right)\right)+h(\ell), \quad \ell \in \mathbb{Z},
$$

where $h(\ell):=\sum_{j=2}^{N} \sum_{n \in \mathbb{Z}} \mathcal{L}_{j}[f]\left(\sigma_{j}+n r\right) \mathcal{L}_{1}\left[S_{j}\right]\left(\sigma_{1}+(\ell-n) r\right)$.

For any fixed $f \in V(\phi)$, set $\mathbf{s}=\{s(n)\}_{n \in \mathbb{Z}}, \mathbf{u}=\{u(n)\}_{n \in \mathbb{Z}}$, and $\mathbf{h}=$ $\{h(n)\}_{n \in \mathbb{Z}}$ where $s(n)=\mathcal{L}_{1}[f]\left(\sigma_{1}+n r\right)$ and $u(n)=\mathcal{L}_{1}\left[S_{1}\right]\left(\sigma_{1}+n r\right)$. Then $(3)$ becomes

$$
s(\ell)=\sum_{n \in \mathbb{Z}} s(n) u(\ell-n)+h(\ell), \quad \ell \in \mathbb{Z},
$$

where finitely many entries $M=\{s(n): n \in I\}=\left\{s\left(n_{1}\right), \cdots, s\left(n_{m}\right)\right\}$ are missing from $\mathbf{s}$.

Theorem 3.3. Assume that $\mathbf{u}$ is one-side supported. Then any finitely many missing entries $\{s(n): n \in I\}$ can be recovered from (4) if and only if $\mathbf{u} \neq \boldsymbol{\delta}_{0}$.

Proof. For $\ell \in I$, (4) becomes

$$
s\left(n_{j}\right)=\sum_{n \in I} s(n) u\left(n_{j}-n\right)+\sum_{n \notin I} s(n) u\left(n_{j}-n\right)+h\left(n_{j}\right), \quad 1 \leq j \leq m
$$


so that

$\sum_{k=1}^{m} s\left(n_{k}\right)\left(\delta_{j, k}-u\left(n_{j}-n_{k}\right)\right)=\sum_{n \notin I} s(n) u\left(n_{j}-n\right)+h\left(n_{j}\right):=g\left(n_{j}\right), \quad 1 \leq j \leq m$.

For $\ell \notin I,(4)$ becomes

$$
-\sum_{k=1}^{m} s\left(n_{k}\right) u\left(\ell-n_{k}\right)=\sum_{n \notin I} s(n) u(\ell-n)+h(\ell)-s(\ell):=g(\ell)
$$

Let $\mathbf{x}=\left[s\left(n_{k}\right)\right]_{k=1}^{m}$ and $\mathbf{g}=[g(\ell)]_{\ell \in \mathbb{Z}}$ be column vectors. Then we can express (5) and (6) in matrix form as

$$
\mathbf{B x}=\mathbf{g}
$$

where $\mathbf{B}=\left[b_{j, k}\right]_{j \in \mathbb{Z}, 1 \leq k \leq m}$ and $b_{j, k}=\delta_{j, n_{k}}-u\left(j-n_{k}\right)$. Note that the $k$-th column of $\mathbf{B}$ is $\mathbf{B}^{k}=\tau_{n_{k}}\left(\boldsymbol{\delta}_{0}-\mathbf{u}\right)=\tau_{n_{k}}\left(\mathbf{u}^{(0)}\right)$, where $\mathbf{u}^{(0)}:=\boldsymbol{\delta}_{0}-\mathbf{u}$, i.e. $\mathbf{B}^{k}$ is a shift of $\mathbf{u}^{(0)}$ by units $n_{k}$.

Assume $\mathbf{u}=\boldsymbol{\delta}_{0}$, i.e. $\mathbf{u}^{(0)}=0$. Then (4) becomes the trivial equation: $s(\ell)=$ $s(\ell), \ell \in \mathbb{Z}$, or equivalently $\mathbf{B}=0$ so no missing entry from $\mathbf{s}$ can be recovered from (4).

Now assume $\mathbf{u} \neq \boldsymbol{\delta}_{0}$, i.e. $\mathbf{u}^{(0)} \neq 0$. Then $\mathbf{B}^{k}=\tau_{n_{k}}\left(\mathbf{u}^{(0)}\right) \neq 0,1 \leq k \leq m$.

First assume $\mathbf{u}$ is upper supported, i.e., $\alpha(\mathbf{u})$ is finite. Then $p:=\alpha\left(\mathbf{u}^{(0)}\right)$ is also finite so that $u^{(0)}(n)=0$ for $n<p$ and $u^{(0)}(p) \neq 0$. Since $\alpha\left(\mathbf{B}^{k}\right)=$ $\alpha\left(\mathbf{u}^{(0)}\right)+n_{k}=p+n_{k}, 1 \leq k \leq m$,

$$
\mathbf{B}_{p+n_{k}}=[*, \cdots, *, \underbrace{u^{(0)}(p)}_{k-t h}, 0, \cdots, 0], \quad 1 \leq k \leq m .
$$

Let $\mathbf{B}_{R}:=\left[\mathbf{B}_{p+n_{k}}\right]_{k=1}^{m}$. Then $\mathbf{B}_{R}$ is a non-singular $m \times m$ lower triangular matrix with $\operatorname{det} \mathbf{B}_{R}=u^{(0)}(p)^{m} \neq 0$ and

$$
\mathbf{B}_{R} \mathbf{x}=\mathbf{g}_{R}:=\left[g\left(p+n_{k}\right)\right]_{k=1}^{m}
$$

from which we can recover $\mathbf{x}$ as $\mathbf{x}=\mathbf{B}_{R}^{-1} \mathbf{g}_{R}$.

Now assume $\mathbf{u}$ is lower supported, i.e., $\beta(\mathbf{u})$ is finite. Then $q:=\beta\left(\mathbf{u}^{(0)}\right)$ is also finite. Then $u^{(0)}(n)=0$ for $n>q$ and $u^{(0)}(q) \neq 0$. Since $\beta\left(\mathbf{B}^{k}\right)=$ $\beta\left(\mathbf{u}^{(0)}\right)+n_{k}=q+n_{k}, 1 \leq k \leq m$,

$$
\mathbf{B}_{q+n_{k}}=[0, \cdots, 0, \underbrace{u^{(0)}(q)}_{k-t h}, *, \cdots, *], \quad 1 \leq k \leq m .
$$

so that $\mathbf{B}_{R}:=\left[\mathbf{B}_{q+n_{k}}\right]_{k=1}^{m}$ is a non-singular $m \times m$ upper triangular matrix with $\operatorname{det} \mathbf{B}_{R}=u^{(0)}(q)^{m} \neq 0$ satisfying (8), where $\mathbf{g}_{R}:=\left[g\left(q+n_{k}\right)\right]_{k=1}^{m}$. Hence again we can recover $\mathbf{x}$ as $\mathbf{x}=\mathbf{B}_{R}^{-1} \mathbf{g}_{R}$. 
It is worth to note that if $r=N$ so that $\left\{S_{j}(t-n r): 1 \leq j \leq N, n \in \mathbb{Z}\right\}$ in $(1)$ is a Riesz basis of $V(\phi)$, then $\mathcal{L}_{j}\left[S_{j}\right]\left(\sigma_{j}+n r\right)=\delta_{0}(n)$ for any $1 \leq j \leq N$ which implies $\mathbf{u}=\boldsymbol{\delta}_{0}$ in Theorem 3.3.

Remark 3.4. In most previous works [2, 3, 4, 8, 11] on recovery of missing samples, one considers only the equation (5), which can be rewritten in a matrix form as

$$
(\mathbf{I}-\mathbf{R}) \mathbf{x}=\mathbf{y}
$$

where $\mathbf{x}=\left[s\left(n_{k}\right)\right]_{k=1}^{m}, \mathbf{y}=\left[g\left(n_{k}\right)\right]_{k=1}^{m}$ and $\mathbf{R}=\left[u\left(n_{j}-u_{k}\right)\right]_{j, k=1}^{m}$, and tries to show that 1 is not an eigenvalue of the interpolation matrix $\mathbf{R}$.

Case 2: finitely many missing samples from two channels We may assume that finitely many samples are missing from the first and second channels, say, $M=\left\{\mathcal{L}_{1}[f]\left(\sigma_{1}+n r\right): n \in I_{1}\right\} \cup\left\{\mathcal{L}_{2}[f]\left(\sigma_{2}+n r\right): n \in I_{2}\right\}$ are missing, where $I_{1}=\left\{n_{1}^{1}<\cdots<n_{m_{1}}^{1}\right\}$ and $I_{2}=\left\{n_{1}^{2}<\cdots<n_{m_{2}}^{2}\right\}\left(m_{1}, m_{2} \geq 1\right)$. As in Case 1 , we have from (2)

$$
\begin{aligned}
\mathcal{L}_{1}[f]\left(\sigma_{1}+\ell r\right)= & \sum_{n \in \mathbb{Z}} \mathcal{L}_{1}[f]\left(\sigma_{1}+n r\right) \mathcal{L}_{1}\left[S_{1}\right]\left(\sigma_{1}+(\ell-n) r\right) \\
& +\sum_{n \in \mathbb{Z}} \mathcal{L}_{2}[f]\left(\sigma_{2}+n r\right) \mathcal{L}_{1}\left[S_{2}\right]\left(\sigma_{1}+(\ell-n) r\right) \\
& +\sum_{j=3}^{N} \sum_{n \in \mathbb{Z}} \mathcal{L}_{j}[f]\left(\sigma_{j}+n r\right) \mathcal{L}_{1}\left[S_{j}\right]\left(\sigma_{1}+(\ell-n) r\right)
\end{aligned}
$$

and

$$
\begin{aligned}
\mathcal{L}_{2}[f]\left(\sigma_{2}+\ell r\right)= & \sum_{n \in \mathbb{Z}} \mathcal{L}_{1}[f]\left(\sigma_{1}+n r\right) \mathcal{L}_{2}\left[S_{1}\right]\left(\sigma_{2}+(\ell-n) r\right) \\
& +\sum_{n \in \mathbb{Z}} \mathcal{L}_{2}[f]\left(\sigma_{2}+n r\right) \mathcal{L}_{2}\left[S_{2}\right]\left(\sigma_{2}+(\ell-n) r\right) \\
& +\sum_{j=3}^{N} \sum_{n \in \mathbb{Z}} \mathcal{L}_{j}[f]\left(\sigma_{j}+n r\right) \mathcal{L}_{2}\left[S_{j}\right]\left(\sigma_{2}+(\ell-n) r\right) .
\end{aligned}
$$

For each $n \in \mathbb{Z}$, set $s_{1}(n)=\mathcal{L}_{1}[f]\left(\sigma_{1}+n r\right), s_{2}(n)=\mathcal{L}_{2}[f]\left(\sigma_{2}+n r\right), u_{11}(n)=$ $\mathcal{L}_{1}\left[S_{1}\right]\left(\sigma_{1}+n r\right), u_{12}(n)=\mathcal{L}_{1}\left[S_{2}\right]\left(\sigma_{1}+n r\right), u_{21}(n)=\mathcal{L}_{2}\left[S_{1}\right]\left(\sigma_{2}+n r\right), u_{22}(n)=$ $\mathcal{L}_{2}\left[S_{2}\right]\left(\sigma_{2}+n r\right), h_{1}(n)=\sum_{j=3}^{N} \sum_{k \in \mathbb{Z}} \mathcal{L}_{j}[f]\left(\sigma_{j}+k r\right) \mathcal{L}_{1}\left[S_{j}\right]\left(\sigma_{1}+(n-k) r\right)$, and $h_{2}(n)=\sum_{j=3}^{N} \sum_{k \in \mathbb{Z}} \mathcal{L}_{j}[f]\left(\sigma_{j}+k r\right) \mathcal{L}_{2}\left[S_{j}\right]\left(\sigma_{2}+(n-k) r\right)$. Then (9) and (10) can be written as

$$
s_{1}(\ell)=\sum_{n \in \mathbb{Z}} s_{1}(n) u_{11}(\ell-n)+\sum_{n \in \mathbb{Z}} s_{2}(n) u_{12}(\ell-n)+h_{1}(\ell), \quad \ell \in \mathbb{Z},
$$


and

$$
s_{2}(\ell)=\sum_{n \in \mathbb{Z}} s_{1}(n) u_{21}(\ell-n)+\sum_{n \in \mathbb{Z}} s_{2}(n) u_{22}(\ell-n)+h_{2}(\ell), \quad \ell \in \mathbb{Z} .
$$

Equation (11) for $\ell \in I_{1}$ and $\ell \notin I_{1}$ become

$\sum_{k=1}^{m_{1}} s_{1}\left(n_{k}^{1}\right)\left(\delta_{j, k}-u_{11}\left(n_{j}^{1}-n_{k}^{1}\right)\right)-\sum_{k=1}^{m_{2}} s_{2}\left(n_{k}^{2}\right) u_{12}\left(n_{j}^{1}-n_{k}^{2}\right)=g_{1}\left(n_{j}^{1}\right), \quad 1 \leq j \leq m_{1}$,

where $g_{1}\left(n_{j}^{1}\right)=\sum_{n \notin I_{1}} s_{1}(n) u_{11}\left(n_{j}^{1}-n\right)+\sum_{n \notin I_{2}} s_{2}(n) u_{12}\left(n_{j}^{1}-n\right)+h_{1}\left(n_{j}^{1}\right)$ and

$$
-\sum_{k=1}^{m_{1}} s_{1}\left(n_{k}^{1}\right) u_{11}\left(\ell-n_{k}^{1}\right)-\sum_{k=1}^{m_{2}} s_{2}\left(n_{k}^{2}\right) u_{12}\left(\ell-n_{k}^{2}\right)=g_{1}(\ell), \quad \ell \notin I_{1}
$$

where $g_{1}(\ell)=\sum_{n \notin I_{1}} s_{1}(n) u_{11}(\ell-n)+\sum_{n \notin I_{2}} s_{2}(n) u_{12}(\ell-n)+h_{1}(\ell)-s_{1}(\ell)$.

Equation (12) for $\ell \in I_{2}$ and $\ell \notin I_{2}$ become

$$
-\sum_{k=1}^{m_{1}} s_{1}\left(n_{k}^{1}\right) u_{21}\left(n_{j}^{2}-n_{k}^{1}\right)+\sum_{k=1}^{m_{2}} s_{2}\left(n_{k}^{2}\right)\left(\delta_{j, k}-u_{22}\left(n_{j}^{2}-n_{k}^{2}\right)\right)=g_{2}\left(n_{j}^{2}\right), \quad 1 \leq j \leq m_{2},
$$

where $g_{2}\left(n_{j}^{2}\right)=\sum_{n \notin I_{1}} s_{1}(n) u_{21}\left(n_{j}^{2}-n\right)+\sum_{n \notin I_{2}} s_{2}(n) u_{22}\left(n_{j}^{2}-n\right)+h_{2}\left(n_{j}^{2}\right)$ and

$$
-\sum_{k=1}^{m_{1}} s_{1}\left(n_{k}^{1}\right) u_{21}\left(\ell-n_{k}^{1}\right)-\sum_{k=1}^{m_{2}} s_{2}\left(n_{k}^{2}\right) u_{22}\left(\ell-n_{k}^{2}\right)=g_{2}(\ell), \quad \ell \notin I_{2}
$$

where $g_{2}(\ell)=\sum_{n \notin I_{1}} s_{1}(n) u_{21}(\ell-n)+\sum_{n \notin I_{2}} s_{2}(n) u_{22}(\ell-n)+h_{2}(\ell)-s_{2}(\ell)$.

Let $\mathbf{x}_{1}=\left[s_{1}\left(n_{k}^{1}\right)\right]_{k=1}^{m_{1}}, \mathbf{x}_{2}=\left[s_{2}\left(n_{k}^{2}\right)\right]_{k=1}^{m_{2}}, \mathbf{x}=\left[\begin{array}{c}\mathbf{x}_{1} \\ \mathbf{x}_{2}\end{array}\right], \mathbf{g}_{1}=\left[g_{1}(n)\right]_{n \in \mathbb{Z}}, \mathbf{g}_{2}=$ $\left[g_{2}(n)\right]_{n \in \mathbb{Z}}$, and $\mathbf{g}=\left[\begin{array}{l}\mathbf{g}_{1} \\ \mathbf{g}_{2}\end{array}\right]$ be column vectors. Then we may express (13)-(16) in matrix form as

$$
A \mathbf{x}=\left[\begin{array}{ll}
\mathbf{B} & \mathbf{C} \\
\mathbf{D} & \mathbf{E}
\end{array}\right] \mathbf{x}=\mathbf{g}
$$

where

$\mathbf{B}=\left[b_{j, k}\right]_{j \in \mathbb{Z}, 1 \leq k \leq m_{1}}=\left[\mathbf{B}^{k}\right]_{1 \leq k \leq m_{1}}$ with $b_{j, k}=\delta_{j, n_{k}^{1}}-u_{11}\left(j-n_{k}^{1}\right), \mathbf{B}^{k}=\tau_{n_{k}^{1}}\left(\boldsymbol{\delta}_{0}-\right.$ $\left.\mathbf{u}_{11}\right)$,

$\mathbf{C}=\left[c_{j, k}\right]_{j \in \mathbb{Z}, 1 \leq k \leq m_{2}}=\left[\mathbf{C}^{k}\right]_{1 \leq k \leq m_{2}}$ with $c_{j, k}=-u_{12}\left(j-n_{k}^{2}\right), \mathbf{C}^{k}=\tau_{n_{k}^{2}}\left(-\mathbf{u}_{12}\right)$, $\mathbf{D}=\left[d_{j, k}\right]_{j \in \mathbb{Z}, 1 \leq k \leq m_{1}}=\left[\mathbf{D}^{k}\right]_{1 \leq k \leq m_{1}}$ with $d_{j, k}=-u_{21}\left(j-n_{k}^{1}\right), \mathbf{D}^{k}=\tau_{n_{k}^{1}}\left(-\mathbf{u}_{21}\right)$, $\mathbf{E}=\left[e_{j, k}\right]_{j \in \mathbb{Z}, 1 \leq k \leq m_{2}}=\left[\mathbf{E}^{k}\right]_{1 \leq k \leq m_{2}}$ with $e_{j, k}=\delta_{j, n_{k}^{2}}-u_{22}\left(j-n_{k}^{2}\right), \mathbf{E}^{k}=\tau_{n_{k}^{2}}\left(\boldsymbol{\delta}_{0}-\right.$ $\left.\mathbf{u}_{22}\right)$. 
Theorem 3.5. Assume $\mathbf{u}_{j}:=\boldsymbol{\delta}_{0}-\mathbf{u}_{j j} \neq 0$ for $j=1,2$. Let $p_{j}:=\alpha\left(\mathbf{u}_{j}\right)$, $q_{j}:=\beta\left(\mathbf{u}_{j}\right)$ and $\Delta_{j}:=\min _{1 \leq k \leq m_{j}-1}\left(n_{k+1}^{j}-n_{k}^{j}\right)$ for $j=1,2$. Then any finitely many missing entries $M=\left\{s_{1}(n): n \in I_{1}\right\} \cup\left\{s_{2}(n): n \in I_{2}\right\}$ can be recovered from (11) and (12) if any one of the following holds:

(i) $\mathbf{u}_{1}$ is upper supported, $\mathbf{u}_{2}$ is one-side supported, and there exists an integer $0 \leq d<\Delta_{1}$ such that $u_{1}\left(p_{1}+d\right) \neq 0$ and $p_{1}+d+n_{k}^{1}-n_{\ell}^{2} \notin \operatorname{supp} \mathbf{u}_{12}$ for $1 \leq k \leq m_{1}, 1 \leq \ell \leq m_{2}$;

(ii) $\mathbf{u}_{1}$ is lower supported, $\mathbf{u}_{2}$ is one-side supported, and there exists an integer $0 \leq d<\Delta_{1}$ such that $u_{1}\left(q_{1}-d\right) \neq 0$ and $q_{1}-d+n_{k}^{1}-n_{\ell}^{2} \notin \operatorname{supp} \mathbf{u}_{12}$ for $1 \leq k \leq m_{1}, 1 \leq \ell \leq m_{2}$;

(iii) $\mathbf{u}_{1}$ is one-side supported, $\mathbf{u}_{2}$ is upper supported, and there exists an integer $0 \leq d<\Delta_{2}$ such that $u_{2}\left(p_{2}+d\right) \neq 0$ and $p_{2}+d+n_{\ell}^{2}-n_{k}^{1} \notin \operatorname{supp} \mathbf{u}_{21}$ for $1 \leq k \leq m_{1}, 1 \leq \ell \leq m_{2}$;

(iv) $\mathbf{u}_{1}$ is one-side supported, $\mathbf{u}_{2}$ is lower supported, and there exists an integer $0 \leq d<\Delta_{2}$ such that $u_{2}\left(q_{2}-d\right) \neq 0$ and $q_{2}-d+n_{\ell}^{2}-n_{k}^{1} \notin \operatorname{supp} \mathbf{u}_{21}$ for $1 \leq k \leq m_{1}, 1 \leq \ell \leq m_{2}$;

(v) $-\infty<\alpha\left(\mathbf{B}^{m_{1}}\right)<\alpha\left(\mathbf{C}^{1}\right)<\infty$;

(vi) $-\infty<\beta\left(\mathbf{B}^{m_{1}}\right)<\beta\left(\mathbf{C}^{1}\right)<\infty$;

(vii) $-\infty<\alpha\left(\mathbf{D}^{m_{1}}\right)<\alpha\left(\mathbf{E}^{1}\right)<\infty$;

(viii) $-\infty<\beta\left(\mathbf{D}^{m_{1}}\right)<\beta\left(\mathbf{E}^{1}\right)<\infty$.

Proof. (i) Assume that $\mathbf{u}_{1}$ and $\mathbf{u}_{2}$ are upper supported (i.e., $p_{1}$ and $p_{2}$ are finite) and there exists an integer $0 \leq d<\Delta_{1}$ such that $u_{1}\left(p_{1}+d\right) \neq 0$ and $p_{1}+d+n_{k}^{1}-n_{\ell}^{2} \notin \operatorname{supp} \mathbf{u}_{12}$ for $1 \leq k \leq m_{1}, 1 \leq \ell \leq m_{2}$. Since $p_{1}+d+$ $n_{k}^{1}<p_{1}+\Delta_{1}+n_{k}^{1} \leq p_{1}+\left(n_{k+1}^{1}-n_{k}^{1}\right)+n_{k}^{1}=p_{1}+n_{k+1}^{1}=\alpha\left(B^{k+1}\right)$ for each $1 \leq k \leq m_{1}-1$, we have

$$
\mathbf{B}_{p_{1}+d+n_{k}^{1}}=[*, \cdots, *, \underbrace{u_{1}\left(p_{1}+d\right)}_{k-t h}, 0, \cdots, 0], \quad 1 \leq k \leq m_{1} .
$$

Then as in the proof of Theorem 3.3, $\mathbf{B}_{R}:=\left[\mathbf{B}_{p_{1}+d+n_{k}^{1}}\right]_{k=1}^{m_{1}}$ and $\mathbf{E}_{R}:=\left[\mathbf{E}_{p_{2}+n_{k}^{2}}\right]_{k=1}^{m_{2}}$ are non-singular lower triangular matrices with $\operatorname{det} \mathbf{B}_{R}=u_{1}\left(p_{1}+d\right)^{m_{1}}$ and $\operatorname{det} \mathbf{E}_{R}=u_{2}\left(p_{2}\right)^{m_{2}}$. On the other hand, $\mathbf{C}_{R}:=\left[\mathbf{C}_{p_{1}+d+n_{k}^{1}}\right]_{k=1}^{m_{1}}=0$ since $c_{p_{1}+d+n_{k}^{1}, \ell}=-u_{12}\left(p_{1}+d+n_{k}^{1}-n_{\ell}^{2}\right)=0$ for $1 \leq k \leq m_{1}, 1 \leq \ell \leq m_{2}$. We then have from (17)

$$
A_{R} \mathbf{x}=\left[\begin{array}{ll}
\mathbf{B}_{R} & \mathbf{C}_{R} \\
\mathbf{D}_{R} & \mathbf{E}_{R}
\end{array}\right] \mathbf{x}=\mathbf{g}_{R}
$$


where $\mathbf{D}_{R}:=\left[\mathbf{D}_{p_{2}+n_{k}^{2}}\right]_{k=1}^{m_{2}}, \mathbf{g}_{R}:=\left[\begin{array}{l}\mathbf{g}_{R}^{1} \\ \mathbf{g}_{R}^{2}\end{array}\right], \mathbf{g}_{R}^{1}:=\left[g_{1}\left(p_{1}+d+n_{k}^{1}\right)\right]_{k=1}^{m_{1}}$, and $\mathbf{g}_{R}^{2}:=\left[g_{2}\left(p_{2}+n_{k}^{2}\right)\right]_{k=1}^{m_{2}}$. Since $\mathbf{C}_{R}=0, \mathbf{A}_{R}$ is a block triangular matrix so that $\operatorname{det} \mathbf{A}_{R}=u_{1}\left(p_{1}+d\right)^{m_{1}} u_{2}\left(p_{2}\right)^{m_{2}} \neq 0$. Hence we can recover $\mathbf{x}$ from (18) as $\mathbf{x}=A_{R}^{-1} \mathbf{g}_{R}$. When $\mathbf{u}_{1}$ is upper supported and $\mathbf{u}_{2}$ is lower supported, the proof is essentially the same as above. The cases (ii), (iii) and (iv) can be also proved similarly as in (i).

(v) Assume $-\infty<\alpha\left(\mathbf{B}^{m_{1}}\right)<\alpha\left(\mathbf{C}^{1}\right)<\infty$ and let $\tilde{p}:=\alpha\left(\mathbf{u}_{12}\right)$. Then $\mathbf{B}_{R}^{1}:=\left[\mathbf{B}_{p_{1}+n_{k}^{1}}\right]_{k=1}^{m_{1}}$ and $\mathbf{C}_{R}^{2}:=\left[\mathbf{C}_{\tilde{p}+n_{k}^{2}}\right]_{k=1}^{m_{2}}$ are non-singular triangular matrices with $\operatorname{det} \mathbf{B}_{R}^{1}=u_{1}\left(p_{1}\right)^{m_{1}}$ and $\operatorname{det} \mathbf{C}_{R}^{2}=\left(-u_{12}(\tilde{p})\right)^{m_{2}}$. Let $\mathbf{C}_{R}^{1}:=\left[\mathbf{C}_{p_{1}+n_{k}^{1}}\right]_{k=1}^{m_{1}}$ and $\mathbf{B}_{R}^{2}:=\left[B_{\tilde{p}+n_{k}^{2}}\right]_{k=1}^{m_{2}}$. Then $\mathbf{C}_{R}^{1}=0$ since $\alpha\left(\mathbf{B}^{m_{1}}\right)=p_{1}+n_{m_{1}}^{1}<\alpha\left(\mathbf{C}^{1}\right)=\tilde{p}+n_{1}^{2}$. We then have from (17)

$$
A_{R} \mathbf{x}=\left[\begin{array}{ll}
\mathbf{B}_{R}^{1} & \mathbf{C}_{R}^{1} \\
\mathbf{B}_{R}^{2} & \mathbf{C}_{R}^{2}
\end{array}\right] \mathbf{x}=\mathbf{g}_{R}
$$

where $\mathbf{g}_{R}=\left[\begin{array}{l}\mathbf{g}_{R}^{1} \\ \mathbf{g}_{R}^{2}\end{array}\right], \mathbf{g}_{R}^{1}=\left[g_{1}\left(p_{1}+n_{k}^{1}\right)\right]_{k=1}^{m_{1}}$, and $\mathbf{g}_{R}^{2}=\left[g_{1}\left(\tilde{p}+n_{k}^{2}\right)\right]_{k=1}^{m_{2}}$. Since $\mathbf{C}_{R}^{1}=0, \mathbf{A}_{R}$ is a block triangular matrix with $\operatorname{det} \mathbf{A}_{R}=u_{1}\left(p_{1}\right)^{m_{1}}\left(-u_{12}(\tilde{p})\right)^{m_{2}} \neq$ 0 . Hence we can recover $\mathbf{x}$ from (19) as $\mathbf{x}=A_{R}^{-1} \mathbf{g}_{R}$. The cases (vi), (vii) and (viii) can be proved similarly as in (v).

In Theorem 3.5 (i)-(iv), if $d=0$, then $u_{1}\left(p_{1}\right) \neq 0$ in (i), $u_{1}\left(q_{1}\right) \neq 0$ in (ii), $u_{2}\left(p_{2}\right) \neq 0$ in (iii), and $u_{2}\left(q_{2}\right) \neq 0$ in (iv) by the definitions of $p_{j}$ and $q_{j}$. Hence we have:

Corollary 3.6. Let $u_{j}, p_{j}$ and $q_{j}$ be the same as in Theorem 3.5. Then any finitely many missing entries $M=\left\{s_{1}(n): n \in I_{1}\right\} \cup\left\{s_{2}(n): n \in I_{2}\right\}$ can be recovered if any one of the following holds:

(i) $\mathbf{u}_{1}$ is upper supported, $\mathbf{u}_{2}$ is one-side supported, and $p_{1}+n_{k}^{1}-n_{\ell}^{2} \notin \operatorname{supp} \mathbf{u}_{12}$ for $1 \leq k \leq m_{1}, 1 \leq \ell \leq m_{2}$;

(ii) $\mathbf{u}_{1}$ is lower supported, $\mathbf{u}_{2}$ is one-side supported, and $q_{1}+n_{k}^{1}-n_{\ell}^{2} \notin \operatorname{supp} \mathbf{u}_{12}$ for $1 \leq k \leq m_{1}, 1 \leq \ell \leq m_{2}$

(iii) $\mathbf{u}_{1}$ is one-side supported, $\mathbf{u}_{2}$ is upper supported, and $p_{2}+n_{\ell}^{2}-n_{k}^{1} \notin \operatorname{supp} \mathbf{u}_{21}$ for $1 \leq k \leq m_{1}, 1 \leq \ell \leq m_{2}$;

(iv) $\mathbf{u}_{1}$ is one-side supported, $\mathbf{u}_{2}$ is lower supported, and $q_{2}+n_{\ell}^{2}-n_{k}^{1} \notin \operatorname{supp} \mathbf{u}_{21}$ for $1 \leq k \leq m_{1}, 1 \leq \ell \leq m_{2}$;

In [8], the same problem of recovering finitely many missing samples from (1) is also considered when the generator $\phi(t)$ and the reconstruction functions $\left\{S_{j}(t)\right\}_{j=1}^{N}$ are compactly supported (see Theorem 3.4 and Theorem 3.5 in [8]). 
In [8], $\mathcal{L}_{j}\left[S_{k}\right](t), 1 \leq j, k \leq N$, are also compactly supported so that the sequences corresponding to $\mathbf{u}$ in Theorem $3.3, \mathbf{u}_{11}, \mathbf{u}_{12}, \mathbf{u}_{21}$, and $\mathbf{u}_{22}$ in Theorem 3.5 are finitely supported. It is clear that Theorem 3.3 above improves Theorem 3.4 in [8]. Also Theorem 3.5 in [8] follows as a consequence of Corollary 3.7 below.

Corollary 3.7. Assume that $\mathbf{u}_{11}, \mathbf{u}_{12}, \mathbf{u}_{21}$, and $\mathbf{u}_{22}$ are finitely supported. For each $j=1,2$, let $p_{j}, q_{j}$ and $\Delta_{j}$ be the same as in Theorem 3.5 and $I^{(j)}=$ $\left[a^{(j)}, b^{(j)}\right]$ be the smallest closed interval containing $\operatorname{supp} \mathbf{u}_{j j}$ where $a^{(j)}$ and $b^{(j)}$ are integers. Then any finitely many missing entries $M=\left\{s_{1}(n): n \in I_{1}\right\} \cup$ $\left\{s_{2}(n): n \in I_{2}\right\}$ can be recovered if any one of the following holds:

(i) $u_{11}(0) \neq 1, \mathbf{u}_{22} \neq \boldsymbol{\delta}_{0}, n_{k}^{1}-n_{\ell}^{2} \notin \operatorname{supp} \mathbf{u}_{12}$ for $1 \leq k \leq m_{1}, 1 \leq \ell \leq m_{2}$, and

$$
\text { either } 0 \notin I^{(1)} \quad \text { or } \quad 2 \Delta_{1}>b^{(1)}-a^{(1)} \text {; }
$$

(ii) $u_{22}(0) \neq 1, \mathbf{u}_{11} \neq \boldsymbol{\delta}_{0}, n_{\ell}^{2}-n_{k}^{1} \notin \operatorname{supp} \mathbf{u}_{21}$ for $1 \leq k \leq m_{1}, 1 \leq \ell \leq m_{2}$, and

$$
\text { either } 0 \notin I^{(2)} \text { or } \quad 2 \Delta_{2}>b^{(2)}-a^{(2)} \text {. }
$$

Proof. Assume that (i) holds. Then $u_{11}(0) \neq 1$ implies that $-\infty<p_{1}=$ $\min \left\{0, a^{(1)}\right\} \leq 0 \leq q_{1}=\max \left\{0, b^{(1)}\right\}<\infty$. If $0 \notin I^{(1)}$, then either $p_{1}=0$ or $q_{1}=0$ so that (i) or (ii) of Theorem 3.5 holds respectively with $d=0$. On the other hand, if $0 \in I^{(1)}$ and $2 \Delta_{1}>b^{(1)}-a^{(1)}$, then $p_{1}=a^{(1)}$ and $q_{1}=b^{(1)}$ so that $2 \Delta_{1}>q_{1}+\left(-p_{1}\right)$, which implies either $\Delta_{1}>-p_{1}$ or $\Delta_{1}>q_{1}$. If $\Delta_{1}>-p_{1}$, then (i) of Theorem 3.5 holds with $d=-p_{1}$. If $\Delta_{1}>q_{1}$, then (ii) of Theorem 3.5 holds with $d=q_{1}$. Hence the claim follows by Theorem 3.5.

Now assume that (ii) holds. Then by similar arguments as above, either (iii) or (iv) of Theorem 3.5 holds so that the claim follows by Theorem 3.5.

We now investigate the case of infinitely many missing samples.

Case 3: infinitely many missing samples from a single channel Assume that infinitely many samples are missing from a single channel, say the first channel $i=1: M=\left\{\mathcal{L}_{1}[f]\left(\sigma_{1}+n \mu r\right): n \in \mathbb{Z}\right\}$ are missing, where $\mu \geq 1$ is an integer. Then with the same notations as in the Case 1, we also have (4) where $M=\{s(\mu n): n \in \mathbb{Z}\}$ are missing.

For $\ell=\mu j, j \in \mathbb{Z}$, (4) becomes

$$
s(\mu j)=\sum_{k \in \mathbb{Z}} s(\mu k) u(\mu(j-k))+\sum_{n \notin \mu \mathbb{Z}} s(n) u(\mu j-n)+h(\mu j)
$$

so that

$$
\sum_{k \in \mathbb{Z}} s(\mu k)\left(\delta_{j, k}-u(\mu(j-k))\right)=g(\mu j)
$$


where $g(\mu j)=\sum_{n \notin \mu \mathbb{Z}} s(n) u(\mu j-n)+h(\mu j)$.

For $\ell \notin \mu \mathbb{Z}$, (4) becomes

$$
-\sum_{k \in \mathbb{Z}} s(\mu k) u(\ell-\mu k)=g(\ell)
$$

where $g(\ell)=\sum_{n \notin \mu \mathbb{Z}} s(n) u(\ell-n)+h(\ell)-s(\ell)$.

Let $\mathbf{x}=[s(\mu k)]_{k \in \mathbb{Z}}$ and $\mathbf{g}=[g(k)]_{k \in \mathbb{Z}}$ be column vectors. Then we can express (20) and (21) in matrix form as

$$
\mathrm{Ax}=\mathrm{g}
$$

where $\mathbf{A}=\left[a_{j, k}\right]_{j, k \in \mathbb{Z}}$ and $a_{j, k}=\delta_{j, \mu k}-u(j-\mu k)$. Decompose the system (22) into $\mu$ subsystems by grouping rows of $A \bmod \mu$ : For $0 \leq \alpha \leq \mu-1$, let

$$
\mathbf{A}^{(\alpha)}:=\left[\mathbf{A}_{\mu j+\alpha}\right]_{j \in \mathbb{Z}} \quad \text { and } \quad \mathbf{g}^{(\alpha)}:=[g(\mu j+\alpha)]_{j \in \mathbb{Z}} .
$$

Then (22) becomes

$$
\mathbf{A}^{(\alpha)} \mathbf{x}=\mathbf{g}^{(\alpha)}, \quad 0 \leq \alpha \leq \mu-1 .
$$

Note that $\mathbf{A}^{(\alpha)}=\left[a_{j, k}^{(\alpha)}\right]_{j, k \in \mathbb{Z}}$ where $a_{j, k}^{(\alpha)}=a_{\mu j+\alpha, k}=a^{(\alpha)}(j-k), a^{(\alpha)}(n)=$ $\delta_{\mu n+\alpha, 0}-u(\mu n+\alpha)$. Hence (23) becomes

$$
\mathbf{a}^{(\alpha)} * \mathbf{x}=\mathbf{g}^{(\alpha)}, \quad 0 \leq \alpha \leq \mu-1 .
$$

Here we note that $\mathbf{a}^{(\alpha)}(0 \leq \alpha \leq \mu-1)$ and $\mathbf{x}$ are in $l^{2}(\mathbb{Z})$ since $\left\{\mathcal{L}_{j}[f]\left(\sigma_{j}+n r\right)\right.$ : $1 \leq j \leq N, n \in \mathbb{Z}\} \in l^{2}(\mathbb{Z})^{N}$ for any $f \in V(\phi)$.

Theorem 3.8. If $0<\left\|\hat{\mathbf{a}}^{(\alpha)}(\xi)\right\|_{0} \leq\left\|\hat{\mathbf{a}}^{(\alpha)}(\xi)\right\|_{\infty}<\infty$ for some $\alpha=0,1, \cdots, \mu-$ 1 , then $M=\left\{\mathcal{L}_{1}[f]\left(\sigma_{1}+n \mu r\right): n \in \mathbb{Z}\right\}$ can be recovered as

$$
\mathcal{L}_{1}[f]\left(\sigma_{1}+n \mu r\right)=\frac{1}{2 \pi} \int_{0}^{2 \pi} \hat{\mathbf{a}}^{(\alpha)}(\xi)^{-1} \hat{\mathbf{g}}^{(\alpha)}(\xi) e^{i n \xi} d \xi, \quad n \in \mathbb{Z} .
$$

Proof. Assume $0<\left\|\hat{\mathbf{a}}^{(\alpha)}(\xi)\right\|_{0} \leq\left\|\hat{\mathbf{a}}^{(\alpha)}(\xi)\right\|_{\infty}<\infty$. Then the convolution operator $\mathbf{a}^{(\alpha)} * \cdot: l^{2}(\mathbb{Z}) \rightarrow l^{2}(\mathbb{Z})$ is an isomorphism with inverse $\mathbf{b}^{(\alpha)} * \cdot$ : $l^{2}(\mathbb{Z}) \rightarrow l^{2}(\mathbb{Z})$, where $\hat{\mathbf{b}}^{(\alpha)}(\xi)=\hat{\mathbf{a}}^{(\alpha)}(\xi)^{-1}$. Hence $(24)$ implies $\mathbf{g}^{(\alpha)} \in l^{2}(\mathbb{Z})$ and $\hat{\mathbf{x}}(\xi)=\hat{\mathbf{a}}^{(\alpha)}(\xi)^{-1} \hat{\mathbf{g}}^{(\alpha)}(\xi)$ from which (25) follows.

If $\mathcal{L}_{j}\left[S_{k}\right](t), 1 \leq j, k \leq N$, are compactly supported, then $\mathbf{a}^{(\alpha)}, 0 \leq \alpha \leq \mu-1$, are finitely supported so that $\hat{\mathbf{a}}^{(\alpha)}(\xi) \in C[0,2 \pi], 0 \leq \alpha \leq \mu-1$. In this case, which happens under the setting of $[8]$, we only require $\left\|\hat{\mathbf{a}}^{(\alpha)}(\xi)\right\|_{0}>0$ for some $\alpha=0,1, \cdots, \mu-1$ in Theorem 3.8 .

As pointed out in [8] and [9], we may allow rational sampling period as well as asymmetric sampling periods in the sampling formula (1). If $r=\frac{p}{q}<1$ where $p$ and $q$ are coprime positive integers, then for each $j=1, \cdots, N$,

$$
\left\{\mathcal{L}_{j}[f]\left(\sigma_{j}+n \frac{p}{q}\right): n \in \mathbb{Z}\right\}=\left\{\mathcal{L}_{j}[f]\left(\sigma_{j}+\frac{p}{q}(k-1)+n p\right): 1 \leq k \leq q, n \in \mathbb{Z}\right\} .
$$


Hence the case of rational sampling period can be reduced to the case of integer sampling period $p$ by extending the number of LTI systems involved.

Case 4: infinitely many missing samples from two channels Assume that infinitely many samples are missing from two channels, say the channels $i=1,2: M=\left\{\mathcal{L}_{1}[f]\left(\sigma_{1}+n \mu r\right): n \in \mathbb{Z}\right\} \cup\left\{\mathcal{L}_{2}[f]\left(\sigma_{2}+n \mu r\right): n \in \mathbb{Z}\right\}$ are missing, where $\mu \geq 1$ is an integer. Then with the same notations as in Case 2, we also have (11) and (12) where $M=\left\{s_{1}(\mu n): n \in \mathbb{Z}\right\} \cup\left\{s_{2}(\mu n): n \in \mathbb{Z}\right\}$ are missing.

Equation (11) for $\ell \in \mu \mathbb{Z}$ and $\ell \notin \mu \mathbb{Z}$ become

$$
\sum_{n \in \mathbb{Z}} s_{1}(\mu n)\left(\delta_{j, n}-u_{11}(\mu(j-n))\right)-\sum_{n \in \mathbb{Z}} s_{2}(\mu n) u_{12}(\mu(j-n))=g_{1}(\mu j), \quad j \in \mathbb{Z}
$$

where $g_{1}(\mu j)=\sum_{n \notin \mu \mathbb{Z}} s_{1}(n) u_{11}(\mu j-n)+\sum_{n \notin \mu \mathbb{Z}} s_{2}(n) u_{12}(\mu j-n)+h_{1}(\mu j)$ and

$$
-\sum_{n \in \mathbb{Z}} s_{1}(\mu n) u_{11}(\ell-\mu n)-\sum_{n \in \mathbb{Z}} s_{2}(\mu n) u_{12}(\ell-\mu n)=g_{1}(\ell), \quad \ell \notin \mu \mathbb{Z}
$$

where $g_{1}(\ell)=\sum_{n \notin \mu \mathbb{Z}} s_{1}(n) u_{11}(\ell-n)+\sum_{n \notin \mu \mathbb{Z}} s_{2}(n) u_{12}(\ell-n)+h_{1}(\ell)-s_{1}(\ell)$.

Let $\mathbf{x}_{1}=\left[s_{1}(\mu n)\right]_{n \in \mathbb{Z}}$ and $\mathbf{x}_{2}=\left[s_{2}(\mu n)\right]_{n \in \mathbb{Z}}$. By an argument similar to Case 3 , we have

$$
\mathbf{a}_{11}^{(\alpha)} * \mathbf{x}_{1}+\mathbf{a}_{12}^{(\alpha)} * \mathbf{x}_{2}=\mathbf{g}_{1}^{(\alpha)}, \quad 0 \leq \alpha \leq \mu-1
$$

where $\mathbf{a}_{11}^{(\alpha)}=\left[a_{11}^{(\alpha)}(n)\right]_{n \in \mathbb{Z}}, \mathbf{a}_{12}^{(\alpha)}=\left[a_{12}^{(\alpha)}(n)\right]_{n \in \mathbb{Z}}$, and $\mathbf{g}_{1}^{(\alpha)}=\left[g_{1}^{(\alpha)}(n)\right]_{n \in \mathbb{Z}}$ with $a_{11}^{(\alpha)}(n)=\delta_{\mu n+\alpha, 0}-u_{11}(\mu n+\alpha), a_{12}^{(\alpha)}(n)=-u_{12}(\mu n+\alpha)$, and $g_{1}^{(\alpha)}(n)=g_{1}(\mu n+$ $\alpha)$.

Let $g_{2}(\mu j)=\sum_{n \notin \mu \mathbb{Z}} s_{1}(n) u_{21}(\mu j-n)+\sum_{n \notin \mu \mathbb{Z}} s_{2}(n) u_{22}(\mu j-n)+h_{2}(\mu j)$ for $j \in \mathbb{Z}$, and $g_{2}(\ell)=\sum_{n \notin \mu \mathbb{Z}} s_{1}(n) u_{21}(\ell-n)+\sum_{n \notin \mu \mathbb{Z}} s_{2}(n) u_{22}(\ell-n)+h_{2}(\ell)-s_{2}(\ell)$ for $\ell \notin \mu \mathbb{Z}$. Then we obtain similarly from (12)

$$
\mathbf{a}_{21}^{(\alpha)} * \mathbf{x}_{1}+\mathbf{a}_{22}^{(\alpha)} * \mathbf{x}_{2}=\mathbf{g}_{2}^{(\alpha)}, \quad 0 \leq \alpha \leq \mu-1
$$

where $\mathbf{a}_{21}^{(\alpha)}=\left[a_{21}^{(\alpha)}(n)\right]_{n \in \mathbb{Z}}, \mathbf{a}_{22}^{(\alpha)}=\left[a_{22}^{(\alpha)}(n)\right]_{n \in \mathbb{Z}}$, and $\mathbf{g}_{2}^{(\alpha)}=\left[g_{2}^{(\alpha)}(n)\right]_{n \in \mathbb{Z}}$ with $a_{21}^{(\alpha)}(n)=-u_{21}(\mu n+\alpha), a_{22}^{(\alpha)}(n)=\delta_{\mu n+\alpha, 0}-u_{22}(\mu n+\alpha)$, and $g_{2}^{(\alpha)}(n)=g_{2}(\mu n+$ $\alpha)$.

We can express (26) and (27) as

$$
H^{(\alpha)}(\xi) \hat{\mathbf{x}}(\xi)=\hat{\mathbf{g}}^{(\alpha)}(\xi)
$$

where $H^{(\alpha)}(\xi):=\left[\begin{array}{ll}\hat{\mathbf{a}}_{11}^{(\alpha)}(\xi) & \hat{\mathbf{a}}_{12}^{(\alpha)}(\xi) \\ \hat{\mathbf{a}}_{21}^{(\alpha)}(\xi) & \hat{\mathbf{a}}_{22}^{(\alpha)}(\xi)\end{array}\right], \hat{\mathbf{x}}(\xi)=\left[\begin{array}{c}\hat{\mathbf{x}}_{1}(\xi) \\ \hat{\mathbf{x}}_{2}(\xi)\end{array}\right]$, and $\hat{\mathbf{g}}^{(\alpha)}(\xi)=\left[\begin{array}{c}\hat{\mathbf{g}}_{1}^{(\alpha)}(\xi) \\ \hat{\mathbf{g}}_{2}^{(\alpha)}(\xi)\end{array}\right]$. 
Theorem 3.9. If $0<\left\|\lambda_{\min }\left(H^{(\alpha)}(\xi)^{*} H^{(\alpha)}(\xi)\right)\right\|_{0} \leq\left\|\lambda_{\max }\left(H^{(\alpha)}(\xi)^{*} H^{(\alpha)}(\xi)\right)\right\|_{\infty}<$ $\infty$ for some $\alpha=0,1, \cdots, \mu-1$, then $M=\left\{\mathcal{L}_{1}[f]\left(\sigma_{1}+n \mu r\right): n \in \mathbb{Z}\right\} \cup\left\{\mathcal{L}_{2}[f]\left(\sigma_{2}+\right.\right.$ $n \mu r): n \in \mathbb{Z}\}$ can be recovered as

$$
\left[\begin{array}{l}
\mathcal{L}_{1}[f]\left(\sigma_{1}+n \mu r\right) \\
\mathcal{L}_{2}[f]\left(\sigma_{2}+n \mu r\right)
\end{array}\right]=\frac{1}{2 \pi} \int_{0}^{2 \pi} H^{(\alpha)}(\xi)^{-1} \hat{\mathbf{g}}^{(\alpha)}(\xi) e^{i n \xi} d \xi, \quad n \in \mathbb{Z} .
$$

Here, $\int_{0}^{2 \pi}\left[a_{j}(\xi)\right]_{j=1,2} d \xi:=\left[\int_{0}^{2 \pi} a_{j}(\xi) d \xi\right]_{j=1,2}$ for $a_{j}(\xi) \in L^{2}[0,2 \pi], j=1,2$.

Proof. Assume $0<\left\|\lambda_{\min }\left(H^{(\alpha)}(\xi)^{*} H^{(\alpha)}(\xi)\right)\right\|_{0} \leq\left\|\lambda_{\max }\left(H^{(\alpha)}(\xi)^{*} H^{(\alpha)}(\xi)\right)\right\|_{\infty}<$ $\infty$. Then the operator $H^{(\alpha)}: L^{2}[0,2 \pi]^{2} \rightarrow L^{2}[0,2 \pi]^{2}$ is bounded invertible a.e. on $[0,2 \pi]$. Hence it follows from $(28)$ that $\left[\begin{array}{l}\hat{\mathbf{x}}_{1}(\xi) \\ \hat{\mathbf{x}}_{2}(\xi)\end{array}\right]=H^{(\alpha)}(\xi)^{-1} \hat{\mathbf{g}}^{(\alpha)}(\xi)$, which implies (29).

Example 3.10. (Band-limited signals) If $\phi(t)=\operatorname{sinc} t:=\frac{\sin \pi t}{\pi t}$, then $V(\phi)=$ $P W_{\pi}$ is the Paley-Wiener space of signals band-limited in $[-\pi, \pi]$. In this case, WSK (Whittaker-Shannon-Kotel'nikov) sampling theorem [7] says that

$$
f(t)=\sum_{n \in \mathbb{Z}} f(n) \operatorname{sinc}(t-n), \quad f(t) \in P W_{\pi}
$$

For any sampling rate $\omega>1$, we also have the oversampling expansion

$$
f(t)=\frac{1}{\omega} \sum_{n \in \mathbb{Z}} f\left(\frac{n}{\omega}\right) \operatorname{sinc}\left(t-\frac{n}{\omega}\right), \quad f \in P W_{\pi}
$$

which converges in $L^{2}(\mathbb{R})$ and absolutely and uniformly on $\mathbb{R}$. Since $\left\{\operatorname{sinc}\left(t-\frac{n}{\omega}\right)\right.$ : $n \in \mathbb{Z}\}$ is an overcomplete frame of $P W_{\pi}$, the redundancy among the samples $\left\{f\left(\frac{n}{\omega}\right): n \in \mathbb{Z}\right\}$ leads to the recovery of finitely many missing samples from (30) $[3,4,11]$.

We now consider the oversampling expansion (30) with a rational sampling rate $\omega=\frac{q}{p}>1$, where $p$ and $q$ are coprime positive integers. Then (30) can be written as

$$
f(t)=\sum_{j=1}^{q} \sum_{n \in \mathbb{Z}} f\left(\frac{p}{q}(j-1)+n p\right) S_{j}(t-n p), \quad f \in P W_{\pi}
$$

where $S_{j}(t)=\frac{p}{q} \operatorname{sinc}\left(t-\frac{p}{q}(j-1)\right)$, which can be viewed as a multi-channel oversampling (1) with $r=p, N=q, l_{j}(t)=\delta(t)$, and $\sigma_{j}=\frac{p}{q}(j-1)$. Note that (31) converges absolutely and uniformly on $\mathbb{R}$ and $\left\{f\left(n \frac{p}{q}\right): n \in \mathbb{Z}\right\} \in l^{2}(\mathbb{Z})$ for any $f \in P W_{\pi}$ by the Plancherel-Pólya inequality (Theorem 6.10 in [7]).

Assume now that infinitely many samples $M=\{f(n p): n \in \mathbb{Z}\}$ are missing from (31). Then we have the equation (22) or equivalently the equation (24) 
with $\mu=1$, where $\mathbf{a}\left(:=\mathbf{a}^{(0)}\right)=\left(1-\frac{p}{q}\right) \boldsymbol{\delta}_{0}, \mathbf{x}=[f(n p)]_{n \in \mathbb{Z}}$ and $\mathbf{g}=[g(n)]_{n \in \mathbb{Z}}$,

$$
g(n)=\frac{p}{q} \sum_{j=2}^{q} \sum_{k \in \mathbb{Z}} f\left(\frac{p}{q}(j-1)+k p\right) \operatorname{sinc}\left((n-k) p-\frac{p}{q}(j-1)\right), \quad n \in \mathbb{Z} .
$$

Since $\hat{\mathbf{a}}(\xi)=1-\frac{p}{q} \neq 0, M$ can be recovered as (cf. (25))

$$
f(n p)=\frac{p}{q-p} \sum_{j=2}^{q} \sum_{k \in \mathbb{Z}} f\left(\frac{p}{q}(j-1)+k p\right) \operatorname{sinc}\left((n-k) p-\frac{p}{q}(j-1)\right), \quad n \in \mathbb{Z} .
$$

\section{ACKNOWLEDGEMENT}

K.H. Kwon and D.G. Lee are partially supported by the National Research Foundation of Korea (NRF) (2012R1A1A2038650). S. Kang is partially supported by EM-BEAM program funded by European Commission.

\section{References}

[1] P. Brianzi and V. Del Prete, Stability of the recovery of missing samples in derivative oversampling, Sampl. Theory Signal Image Process., 10, 191-210, 2011 .

[2] V. Del Prete, Recovery of missing samples in oversampling formulas for band limited functions, Sampl. Theory Signal Image Process., 8, 161-180, 2009 .

[3] P.J.S.G. Ferreira, Incomplete sampling series and the recovery of missing samples from oversampled band-limited signals, IEEE Trans. Signal Process., 40, 225-227, 1992.

[4] P.J.S.G. Ferreira, The stability of a procedure for the recovery of lost samples in band-limited signals, Signal Process., 40, 195-205, 1994.

[5] A.G. García, J.M. Kim, K.H. Kwon, and G.J. Yoon, Multi-channel sampling on shift-invariant spaces with frame generators, Int. J. Wavelets Multiresolut. Inf. Process., 10, DOI: 10.1142/S0219691311004456, 2012.

[6] A.G. García, G. Pérez-Villarón, Dual frames in $L^{2}(0,1)$ connected with generalized sampling in shift-invariant spaces, Appl. Comput. Harmon. Anal., 20, 422-433, 2006.

[7] J.R. Higgins, Sampling Theory in Fourier and Signal Analysis : Foundations, Oxford Univ. Press, Oxford, 1996. 
[8] S. Kang and K.H. Kwon, Recovery of missing samples for oversampling in shift invariant spaces, J. Math. Anal. Appl., 391, 139-146, 2012.

[9] S. Kang, J.M. Kim, and K.H. Kwon, Asymmetric multi-channel sampling in shift invariant spaces, J. Math. Anal. Appl., 367, 20-28, 2010.

[10] J.M. Kim and K.H. Kwon, Sampling expansion in shift invariant spaces, Int. J. Wavelets Multiresolut. Inf. Process., 6, 223-248, 2008.

[11] R.J. Marks II, Introduction to Shannon Sampling and Interpolation Theory, Springer-Verlag, New York, 1991.

[12] A. Ron and Z. Shen, Frames and stable bases for shift-invariant subspaces of $L^{2}\left(\mathbb{R}^{d}\right)$, Canad. J. Math., 47, 1051-1094, 1995.

[13] D.M.S. Santos and P.J.S.G. Ferreira, Reconstruction from missing function and derivative samples and oversampled filter banks, Proc. of the IEEE Int. Conf. on Acoustics, Speech, and Signal Process. ICASSP 2004, 3, 941-944, 2004. 